\title{
The role of human milk oligosaccharides in preventing necrotising enterocolitis and human immunodeficiency virus transmission
}

\author{
Van Niekerk E, BSc(Dietetics), M(Dietetics), Lecturer, Stellenbosch University; Blaauw R, PhD(Nutrition), Associate Professor \\ Division Human Nutrition, Faculty of Medicine and Health Sciences, Stellenbosch University \\ Kirsten GF, MBChB, MMed(Paed), DCH(SA), FCP(Paed)(SA), MD, Professor \\ Department of Paediatrics and Child Health, Division of Neonatology, Faculty of Medicine and Health Sciences, Stellenbosch University \\ Correspondence to: Evette van Niekerk, e-mail: evettev@sun.ac.za \\ Keywords: human immunodeficiency virus, human milk oligosaccharides, necrotising enterocolitis
}

\begin{abstract}
The heavy burden of maternal human immunodeficiency virus (HIV) infection has resulted in a high prevalence of premature births, with an associated increase in the incidence of necrotising enterocolitis (NEC). NEC is a life-threatening inflammatory disease of the gastrointestinal tract that predominantly occurs in preterm infants. Human milk oligosaccharides (HMOs) are carbohydrate compounds which have been shown to have health-promoting effects through their bifidogenic and antiadhesive properties. There is a reduced incidence of NEC in infants who receive human milk, compared to those receiving infant formula. It is suggested that the oligosaccharides found within human milk may act as specific substrates in assisting the growth of selected beneficial bacteria, called probiotics. Probiotics are live microbial food ingredients which have been shown to have health-promoting effects. Lactobacillus rhamnosus GG and Bifidobacterium infantis have been used as probiotics to reduce the incidence of NEC. Furthermore, HMOs have been associated with protection against postnatal HIV transmission. HMOs may hold key responsibility for the prevention of NEC, and possibly the transmission of HIV, to infants in resource-limited settings and in a developing country, such as South Africa, where HIV plays a major role in the outcomes of preterm neonates.
\end{abstract}

(P) Peer reviewed. (Submitted: 2013-07-11. Accepted: 2013-11-23.) ๑ SAJCN

S Afr J Clin Nutr 2014;27(2):51-55

\section{Introduction}

Research, started over a century ago, on human milk oligosaccharides (HMOs) has focused predominantly on their prebiotic effects. Today it is known that the bifidogenic effects of HMOs are one of the many beneficial effects now being discovered. ${ }^{1}$ In addition to providing a substrate for the growth of beneficial bacteria, HMOs also directly inhibit pathogens from adhering to the intestinal epithelia through their antiadhesive properties. ${ }^{1}$ Recent data suggest that firstly, a relationship exists between the HMOs and the incidence of necrotising enterocolitis (NEC), ${ }^{2,3}$ and secondly, they may have an influence on human immunodeficiency virus (HIV) transmission. ${ }^{4}$ This article aims to describe the prebiotic and antiadhesive effects of HMOs, and how they could possibly prevent the development of NEC in the presence of HIV infection.

\section{Human milk oligosaccharides}

$\mathrm{HMOS}^{5,6}$ are complex carbohydrates that are highly abundant in breast milk, but not in infant formula. ${ }^{7}$ Oligosaccharides are carbohydrates made up of 3-9 monosaccharide units. ${ }^{5,6}$ The oligosaccharide concentration of human milk varies with gestational age, lactation duration, and from one mother to another. ${ }^{6,8} \mathrm{HMOS}$ reach the highest concentration in colostrum (20-25 g/l), and then decrease after roughly two weeks to approximately 5-20 g/l in mature breast milk. ${ }^{1,6,8-11}$

HMOs consists of five different monosaccharide units:

- Glucose.

- Galactose.

- The sialic acid component, known as $\mathrm{N}$-acetylglucosamine.

- Fucose.

- $\mathrm{N}$-acteylneuaminic acid. ${ }^{1,12}$

With a few exceptions, all HMOs contain lactose at their reducing end. They may be elongated (an enzymatic attachment) by the addition of $\beta 1-3-$ or $\beta 1-6$-linked lacto-N-biose or $\mathrm{N}$-acetyllacetosamine. The latter can be further extended by one or two disaccharides. Chain branching may occur at the $\beta 1-6$ linkage. Branched lactose molecules may form both linear (para-HMOs) and branched structures (iso-HMOs). Furthermore, oligosaccharide chains can be fucosylated (also known as neutral oligosaccharides) in the $\alpha 1-2, \alpha 1-3$ or $\alpha 1-4$ linkage, or sialylated (also known as acidic oligosaccharides) in the $\alpha 2-3$ or $\alpha 2-6$ linkage. The ABH secretor (Se) status and Lewis (Le) blood group determines the HMO composition of a mother's breast milk. ${ }^{1}$ The presence or absence of blood group substances $(A, B$ and $H)$ in the saliva and other body secretions is controlled by a gene on chromosome 19 , the fucosyltransferase 
locus 2 and the fucosyltransferase locus $3 .{ }^{13}$ Both genes are expressed in glandular epithelia and have dominant allele ( $L e$ and $S e$, respectively) coding for enzymes with fucosyltransferase activity and recessive alleles (le and se, respectively) that are not functional. Briefly, the fucosyltransferase encoded by the $S e$ gene regulates the synthesis of HMOs containing fucose with an $\alpha-1,2$ linkage. The $L e$ gene promotes the synthesis of $\alpha-1,3$ and $\alpha-1,4$ linkages. ${ }^{1}$ There are up to 200 identified HMOs and their effects appear to be strongly structure dependent. 8,11

An atypical characteristic of HMOs is that the monosaccharides, of which they are comprised, are bound by specific bonds resistant to the digestive enzymes present in the small intestine ${ }^{8,14}$ Although intact HMOs may be absorbed, the majority of oligosaccharides ingested through breast milk pass through the small intestine undigested, and reach the colon where they act as a fuel source for bifidus-predominant bacteria, thereby gaining the status of prebiotics. ${ }^{1,15,16}$ A small percentage of absorbed HMOs are excreted in the urine, ${ }^{8,11}$ and may protect the infant from urinary tract infections. ${ }^{17}$ High proportions of bifidobacteria are represented in the intestinal tract of breastfed infants, and through the generation of short-chain fatty acids, they protect the enterocyte from pathogens, and may contribute to mucosal integrity. $8,11,17$

HMO functionality is not limited to their prebiotic effects only. The possibility was raised more than a decade ago that HMOs may act as an anti-infective agent by blocking pathogen adhesion to the intestinal epithelial cell walls, thereby possibly preventing enteric infections, such as rotavirus diarrhoea and systemic infections, such as HIV/acquired immune deficiency syndrome. ${ }^{17}$ The anti-infective effect that they hold has generated considerable interest. The bifidogenic and anti-adhesive properties of HMOs, as well as their role in NEC and the possible linkage to HIV, are now be described in further detail.

\section{The bifidogenic (prebiotic) effect of human milk oligosaccharides}

Factors that influence gastrointestinal tract colonisation can be grouped into two categories: extrinsic and intrinsic. Intrinsic factors include underlying neonatal health, immunological status, gastrointestinal transit time and $\mathrm{pH}$. These factors affect the process of colonisation and the types of organisms established in the neonatal intestinal tract. Extrinsic factors include the geographical area, maternal and surrounding environment bacteria, mode of delivery, feeding practices and drug therapies. ${ }^{18}$

It is well established that the type of delivery has a significant influence on the development of intestinal microflora. ${ }^{19,20}$ Infants born by vaginal delivery are more exposed to their mother's microbiota than those born by Caesarean section. ${ }^{19}$ Initial exposure to microflora by those born by Caesarean delivery is most likely to be from environmental micro-organisms on the equipment, in the air and on other neonates, with the nursing personnel acting as carriers. ${ }^{20}$
Furthermore, the preterm infant is exposed to bacteria in the neonatal intensive care unit, where the frequent use of broad-spectrum antibiotics affects the colonisation of health-promoting bacteria. ${ }^{21}$ The choice of feed plays an important role in the establishment of intestinal flora. Bifidobacterium is a predominant organism found in the intestinal tract of breastfed infants. Lactobacillus and Streptococcus are found in lesser quantities. By contrast, similar amounts of bacteroides and bifidobacterium are found in formula-fed infants, as well as components of the more pathogenic species, such as Staphylococcus, Escherichia coliand Clostridia. ${ }^{21}$ An extraordinary characteristic of the intestinal environment of breastfed infants is the selective nourishment and protective support provided by the intestinal microbiotia. ${ }^{22}$

The term "prebiotic" was introduced and defined by Gibson and Roberfroid as "a non-digestible food ingredient that beneficially affects the host by selectively stimulating the growth and/or activity of one or a limited number of bacteria in the colon" ${ }^{23,24}$ Although HMOs fully conform to this definition, it is important to bear in mind that HMOs promote the growth of certain, but not all, healthpromoting bacteria. ${ }^{1}$ Glycoprofiling results by LoCascio et $\mathrm{al}^{22}$ provide molecular and mechanistic evidence that specific strains of bifidobacteria have an affinity for selected classes of HMO ${ }^{8,11}$ This suggests that the diversity of specific oligosaccharide structures found within human milk do not provide a direct source of nutrition to the infant, but rather act as specific substrates to assist the growth of selected beneficial bacteria, that in their own right support the development and protection of the neonate. ${ }^{22} \mathrm{~N}$-acetylglucosamine oligosaccharides have been implicated as important cell wall components for bifidobacteria. ${ }^{17}$ In the case of $B$. longum by infantis, this strain has the ability to preferentially consume the most abundant HMOs. ${ }^{11,22}$

\section{Antiadhesive effect of human milk oligosaccharides}

There is increasing evidence that oligosaccharides in human milk have a direct inhibitory effect on certain virulence capabilities of pathogenic microorganisms. ${ }^{11}$ The destructiveness of most pathogenic microorganisms, e.g. Campylobacter jejuni, E. coli and Salmonella strains, often depends on their aptitude to adhere to epithelial surfaces. ${ }^{25}$ In general, bacterial adhesion is a ligandreceptor interaction between structures on the bacterial surface and complementary structures on the mucosal surface of the host. ${ }^{11}$

HMOs structurally imitate epithelial cell surface carbohydrates, and therefore act as decoys to which infectious agents can bind, instead of to the host, thereby preventing possible infection. ${ }^{26,27}$ These molecules can withstand the low $\mathrm{pH}$ of the stomach, and resist degradation through enzymes from the pancreas and brush border membrane. ${ }^{28}$ With every feed, the gut of breastfed infants is flushed with various oligosaccharides that can effectively reach and block binding sites, thereby reducing the chances of probable pathogen adhesion to these locations. ${ }^{26}$ 
Human immunodeficiency virus and human milk oligosaccharides

Breastfeeding is the predominant postnatal transmission route for HIV-1 infection in children. ${ }^{29}$ However, the majority of breastfed infants do not become HIV-infected, despite continuous exposure to the virus through their mothers' milk over many months. This raises the question: Does human milk have properties that protect the infant against HIV transmission?

Dendritic cell-specific intercellular adhesion molecule-3-grabbing nonintegrin (DC-SIGN)-bound pathogens are usually transferred into dendritic cell (DC) lysosomes, where they are processed before stimulating T cells to initiate an immune response. ${ }^{25}$ However, HIV-1 pathogens are kept hidden within the $\mathrm{DC}$ for a number of days without prompting an immune response before they are transferred to CD4+ $T$ lymphocytes, where they multiply and cause disease at a later stage. ${ }^{11,30} \mathrm{DC}$-SIGN is a carbohydrate-binding protein. It recognises mannose-containing glycoconjugates, such as HIV-1-gp120, with an even higher binding affinity for $L e$ blood group antigens. ${ }^{25,29,31}$ Although monomeric $L e$ epitopes bind to DC-SIGN, the presence of multivalent $L e$ epitopes is required to compete with HIV-1-gp120 for DC-SIGN-binding. The occurrence of multiple $L e$ blood group determinants as part of HMOs led scientists to postulate that HMOS compete with HIV-1-gp120 for binding to DC-SIGN, and therefore play a protective role against HIV-1 mother-to-child transmission during breastfeeding. ${ }^{1,25}$ Since some HMOs structurally resemble the Le blood group antigens that show high-binding affinity for DCSIGN, and some HMOs carry one or multiple Le epitopes, HMOS may compete with gp120 for binding to DC-SIGN and reduce HIV-1 mother-to-child transmission. ${ }^{31,32}$

However, the results by Bode et al suggest otherwise. ${ }^{4}$ They found that the concentration of HMOs with secretor or $L e$-active epitopes did not correlate with HIV transmission risk. These results propose that the protection against postnatal HIV transmission is probably independent of the mother's secretor or $L e$ blood group status, and that HMOs offer other protective properties, i.e. concentrations of lacto-N-neotetraose. ${ }^{4}$ As described, more than 200 different HMOs have been characterised, and investigators have speculated whether or not the amount will rise to 1000 . Hong et al described the blocking of the DC-SIGN as a two-edged sword, indicating that although it may reduce the entrance of certain viruses, such as HIV-1, at the same time it may also reduce the ability of the infant's immune system to detect and fight other pathogens, leading to a potentially increased risk of bacterial or viral gastroenteritis. ${ }^{29}$ Hong et al have shown that physiological concentrations of HMOS significantly reduce HIV-1gp120-binding to DC-SIGN. ${ }^{25,29}$ Therefore, the question is: Which HMOs are the most potent inhibitors of HIV-1-gp120-DCSIGN binding? ${ }^{29}$ Once individual HMOs have been identified that block HIV-1-gp120 binding to DC-SIGN, it will be important to assess whether or not these HMOs trigger adverse effects. ${ }^{25}$

\section{Necrotising enterocolitis}

NEC is the most common serious acquired condition of the gastrointestinal tract in preterm infants. ${ }^{33}$ NEC occurs in $4-11 \%$ of all premature infants born with a very low birthweight, and an inverse relationship exists between birthweight, gestational age and the incidence of NEC. ${ }^{33,34}$ Generally, prematurity, enteral feeding, ischaemia and bacterial colonisation are the most common risk factors for the development of NEC. ${ }^{35-38}$ The pathogenesis of NEC in neonates born to HIV-infected mothers has not been clearly elucidated. Some described contributing factors include maternal antiretroviral treatment, maternal sepsis and immunological abnormalities. Although antiretroviral prophylaxis has decreased the perinatal transmission of HIV, the burden of NEC in the HIV-exposed newborn population is significant. ${ }^{39,40}$ Infants who receive human milk show a reduced incidence of NEC, compared to those receiving infant formula. ${ }^{41}$ Numerous advantages of breast milk have been found over infant formula. These include the earlier achievement of full enteral feeding, the presence of active enzymes which enhance the maturation of the immature intestinal tract, and anti-infective agents which protect the neonate from infections. ${ }^{42}$ The effect of prebiotics on NEC has not been studied partly because of the very large sample sizes that are required, and secondly because of ethical concerns with such trials.

Jantscher-Krenn et al found that disialyllacto-N-tetraose (DSLNT) has a protective effect against the development of NEC in neonatal rats. ${ }^{2}$ Results by Van Niekerk et al ${ }^{3}$ indicate that DSLNT may indeed protect against NEC, not only in neonatal rats as indicated by Jantscher-Krenn et al, but also in human preterm infants. These results suggest that infants diagnosed with NEC received breast milk that contained lower concentrations of DSLNT than infants who did not develop NEC. Specifically designed and well powered cohort studies are required to verify the link between DSLNT and NEC. If confirmed, DSLNT concentrations in mothers' milk could serve as a noninvasive marker to determine whether or not a breastfed infant is at risk of developing NEC. ${ }^{3}$

\section{The role of probiotics in the management of necrotising enterocolitis}

When focusing on bacterial colonisation, which plays a pivotal role in the development of NEC, ${ }^{43,44}$ it is important to note that no single bacterial species has thus far been associated with NEC. Although Enterobacteriaceae spp. is the most frequently pronounced bacteria to be found in association with NEC, Clostridia spp. and Staphylococcus spp. have also been isolated. ${ }^{45}$ Probiotics might be beneficial in preventing the overgrowth of pathogenic organisms, increased feeding tolerance, ${ }^{46,47}$ decreasing the number of days until full feeds ${ }^{48}$ are reached and preventing nosocomial infections. Furthermore, probiotics potentially compete with other organisms for binding sites and substrate in the bowel. ${ }^{49,50}$ Microbiota disturbances early in HIV infection lead to greater dominance of potential pathogens, reduced levels of Bifidobacteria and Lactobacillus spp. and increasing mucosal inflammation. ${ }^{51}$ As HMOS support the growth of these beneficial bacteria, it is therefore proposed that in conjunction with the use of probiotics, HMOs could potentially reduce the risk of NEC. A randomised clinical trial by van Niekerk et al was the first to examine the use of probiotics in the management of NEC in HIV-exposed versus non-exposed, very low-birthweight 
infants. ${ }^{52}$ The results from this trial concluded that the incidence of death and NEC did not differ significantly between the HIV-exposed and unexposed groups. However, a reduced severity of disease per Bell's staging criteria was found. A significantly lower incidence of NEC was found in the probiotic group than in the control group. ${ }^{52}$

Because the pathogenesis of NEC involves various complex pathways, different probiotic strains may create benefit through different pathways. Evidence suggests that the use of a multistrain or multispecies probiotic should be more effective and more consistent than that of a monostrain probiotic. ${ }^{53}$ Randomised controlled trials that used a multistrain probiotic reported a significant decline in NEC whereas those reporting a lesser decline used a single organism, such as L. rhamnosus GG. ${ }^{49,53,54}$

\section{Conclusion}

HM0s not only act as a substrate for beneficial bacteria, but also provide antiadhesive effects, thereby reducing the risk of infection. ${ }^{1}$ Many unanswered questions remain that surround the mother-tochild transmission of HIV and the role of HMOs. Furthermore, the incidence of NEC in premature infants has increased as the burden of HIV has heightened. ${ }^{55}$ Therefore, the question remains: Could HMOs serve as a prophylactic measure against this life-threatening gastrointestinal condition? Further studies are needed to investigate the influence of HMOs on the attachment of pathogens to the intestinal epithelial cell walls. To fully understand the immunological needs of the preterm infant, HMO concentrations in preterm breast milk need to be clearly defined. Future prospects for HMO research are expected to be very useful with regard to the development of management options that are effective in protecting babies from harmful infections.

Breast milk is widely recognised as the optimal feed of choice for both term and preterm infants, as it upholds metabolic, immunological and neurodevelopmental advantages..$^{56}$ The World Health Organization ${ }^{57}$ recommends exclusive breastfeeding for all infants, and recommends modifications to breastfeeding, such as breast milk expression and pasteurisation, to reduce the risk of HIV transmission, while providing the advantages of breast milk's immune-stimulating properties. ${ }^{58}$ The use of breast milk should be strongly advocated in neonatal units. Postnatal breastfeeding practices in resource-limited settings and developing countries such as South Africa, can be supported by practising kangaroo mother care in neonatal units. A 2012 Cochrane review concluded that kangaroo mother care improves anthropometric parameters, breastfeeding and mother satisfaction. ${ }^{59}$ Mothers of preterm infants should be encouraged to express their breast milk and pasteurise it, if applicable. Breastfeeding must also be enforced when it is physiologically possible for preterm infants.

\section{Acknowledgements}

We would like to thank Prof Peter Donald for his thoughtful review of the manuscript.

\section{References}

1. Bode L. Human milk oligosaccharides: every baby needs a sugar mama. Glycobiology. 2012;22(9):1147-1162.

2. Jantscher-Krenn E, Zherebtsov M, Nissan C, et al. The human milk oligosaccharide disialyllacto-N-tetraose prevents necrotising enterocolitis in neonatal rats. Gut. 2012;61(10):1417-1425.

3. Van Niekerk E, Autran CA, Nel DG, et al. Human milk oligosaccharides differ between HIVinfected and -uninfected mothers and are related to necrotizing enterocolitis incidence in their preterm very low birthweight infants. J Nutr. 2014 [Epub ahead of print].

4. Bode L, Kuhn L, Kim H-Y, et al. Human milk oligosaccharide concentration and risk of postnatal transmission of HIV through breastfeeding. Am J Clin Nutr. 2012;96(4):831-839.

5. Yang B, Chuang H, Chen R-F. Protection from viral infections by human milk oligosaccharides: direct blockade and indirect modulation of intestinal ecology and immune reactions. Open Glycoscience. 2012;5:19-25

6. Coppa GV, Bruni S, Morelli L, et al. The first prebiotics in humans: human milk oligosaccharides. J Clinical Gastroenterol. 2004;38(6 Suppl):S80-S83.

7. Vandenplas Y. Oligosaccharides in infant formula. Br J Nutr. 2002;87(2):293-296.

8. Chichlowski M, German JB, Lebrilla CB, Mills DA. The influence of milk oligosaccharides on microbiota of infants: opportunities for formulas. Annu Rev Food Sci Technol. 2011;2:331-351.

9. Coppa GV, Gabrielli 0 , Pierani $P$, et al. Changes in carbohydrate composition in human milk over 4 months of lactation. Pediatrics. 1993;91(3):637-641.

10. Coppa G, Pierani P, Zampini L, et al. Oligosaccharides in human milk during different phases of lactation. Acta Paediatr Suppl. 1999;88(430):89-94

11. Kunz C, Rudloff S. Biological functions of oligosaccharides in human milk. Acta Paediatr 1993;82(12):903-912

12. McVeagh P, Miller JB. Human milk oligosaccharides: only the breast. J Paediatr Child Health. 1997;33(4):281-286.

13. Gloria-Bottini F, Magrini A, Cozzoli E, et al. ABH secretor genetic polymorphism: evidence of intrauterine selection. Eur J Obstet Gynecol Reprod Bio. 2011;154(1):20-23.

14. Chaturvedi $P$, Warren $C D$, Buescher CR, et al. Survival of human milk oligosaccharides in the intestine of infants. Adv Exp Med Biol. 2001;501:315-323.

15. Coppa G, Zampini L, Galeazzi T, Gabrielli 0. Prebiotics in human milk: a review. Dig Liver Dis. 2006;38 Suppl 2:S291-S294.

16. Engfer MB, Stahl B, Finke B, et al. Human milk oligosaccharides are resistant to enzymatic hydrolysis in the upper gastrointestinal tract. Am J Clin Nutr. 2000;71(6):1589-1596.

17. Uauy $R$, Araya M. Novel oligosaccharides in human milk: understanding mechanisms may lead to better prevention of enteric and other infections. J Pediatr. 2004;145(3):297-303

18. Holzapfel WH, Haberer P, Snel J, Schillinger U. Overview of gut flora and probiotics. Int J Food Microbiol. 1998:41(2):85-101.

19. Biasucci G, Benenati B, Morelli L, et al. Cesarean delivery may affect the early biodiversity of intestinal bacteria. J Nutr. 2008;138(9):1796S-1800S.

20. Mackie RI, Sghir A, Gaskins HR. Developmental microbial ecology of the neonatal gastrointestinal tract. Am J Clin Nutr. 1999;69(5):1035S-1045S

21. Claud EC, Walker WA. Hypothesis: inappropriate colonization of the premature intestine can cause neonatal necrotizing enterocolitis. The FASEB Journal. 2001;15(8):1398-1403

22. LoCascio RG, Ninonuevo MR, Freeman SL, et al. Glycoprofiling of bifidobacterial consumption of human milk oligosaccharides demonstrates strain specific, preferentia consumption of small chain glycans secreted in early human lactation. J Agricult Food Chem. 2007;55(22):8914-8919.

23. Schrezenmeir J, de Vrese M. Probiotics, prebiotics, and synbiotics: approaching a definition. Am J Clin Nutr. 2001;(2 Suppl):361S-364S

24. Gibson GR, Roberfroid MB. Dietary modulation of the human colonie microbiota: introducing the concept of prebiotics. J Nutr. 1995;125(6):1401-1412

25. Bode L. Human milk oligosaccharides: prebiotics and beyond. Nutr Rev. 2009;67 Supp 2):S183-S191.

26. Kunz C, Rudloff S, Baier W, et al. Oligosaccharides in human milk: structural, functional, and metabolic aspects. Ann Rev Nutr. 2000;20(1):699-722.

27. Barile D, Rastall RA. Human milk and related oligosaccharides as prebiotics. Curr Opin Biotechnol. 2013;24(2):214-219

28. Bode L. Recent advances on structure, metabolism, and function of human milk oligosaccharides. J Nutr. 2006;136(8):2127-2130.

29. Hong P, Ninonuevo MR, Lee B, et al. Human milk oligosaccharides reduce HIV-1-gp120 binding to dendritic cell-specific ICAM3-grabbing non-integrin (DC-SIGN). Br J Nutr. 2009;101(4):482-486.

30. Van Kooyk Y, Geijtenbeek TB. DC-SIGN: escape mechanism for pathogens. Nat Rev Immunol. 2003;3(9):697-709

31. Hickey RM. The role of oligosaccharides from human milk and other sources in prevention of pathogen adhesion. Int Dairy J. 2012;22(2):141-146.

32. Naarding MA, Ludwig IS, Groot $F$, et al. Lewis $X$ component in human milk binds DC-SIGN and inhibits HIV-1 transfer to CD4+ T lymphocytes. J Clin Invest 2005;115(11):3256-3264 
33. Lee JS, Polin RA. Treatment and prevention of necrotizing enterocolitis. Semin Neonatol. 2003;8(6):449-459.

34. Mihatsch WA. What is the power of evidence recommending routine probiotics for necrotizing enterocolitis prevention in preterm infants? Curr Opin Clin Nutr Metabol Care. 2011;14(3):302-306

35. Lee JS, Polin RA. Treatment and prevention of necrotizing enterocolitis. Semin Neonatol. 2003;8(6):449-459.

36. Lin HC, Su BH, Chen AC, et al. Oral probiotics reduce the incidence and severity of necrotizing enterocolitis in very low birth weight infants. Pediatrics. 2005;115(1):1-4

37. Pellegrini M, Lagrasta N, Garcia CG, et al. Neonatal necrotizing enterocolitis: a focus on Eur Rev Med Pharmacol Sci. 2002;6(1):19-25.

38. Schanler R. Probiotics and necrotising enterocolitis in premature infants. Arch Dis Child Fetal Neonatal Ed. 2006;91(6):F395-F397.

39. Hunter C, Chokshi N, Ford H. Evidence vs experience in the surgical management of necrotizing enterocolitis and focal intestinal perforation. J Perinatol. 2008;28 Supp 1:S14-S17.

40. Karpelowsky JS, van Mil S, Numanoglu A, et al. Effect of maternal human immunodeficiency virus status on the outcome of neonates with necrotizing enterocolitis. J Pediatr Surg. 2010;45(2):315-318

41. Arnold M, Moore SW. HIV exposure does not worsen outcome in stage III necrotizing enterocolitis with current treatment protocols. J Pediatr Surg. 2012;47(4):665-672.

42. McGuire W, Anthony M. Donor human milk versus formula for preventing necrotising enterocolitis in preterm infants: systematic review. Arch Dis Child Fetal Neonatal Ed. 2003;88(1):F11-F14

43. Boyd CA, Quigley MA, Brocklehurst P. Donor breast milk versus infant formula for preterm infants: systematic review and meta-analysis. Arch Dis Child Fetal Neonatal Ed. 2007;92(3):F169-F175

44. Foglia RP. Necrotizing enterocolitis. Curr Probl Surg. 1995;32(9):757-823.

45. Schwiertz A, Gruhl B, Löbnitz M, et al. Development of the intestinal bacterial composition in hospitalized preterm infants in comparison with breast-fed, full-term infants. Pediat Res. 2003;54(3):393-399

46. Hunter CJ, Upperman JS, Ford HR, Camerini V. Understanding the susceptibility of the premature infant to necrotizing enterocolitis (NEC). Pediatr Res. 2008;63(2):117-123.
47. Indrio F, Riezzo G, Raimondi F, et al. The effects of probiotics on feeding tolerance, bowel habits, and gastrointestinal motility in preterm newborns. J Pediatr. 2008;152(6):801-806.

48. Lee SJ, Cho SJ, Park EA. Effects of probiotics on enteric flora and feeding tolerance in preterm infants. Neonatol. 2006;91(3):174-179.

49. AlFaleh K, Anabrees J, Bassler D, Al-Kharfi T. Probiotics for prevention of necrotizing enterocolitis in preterm infants. Cochrane Rev J. 2012;7(6):1807-1854.

50. Mukai T, Asasaka T, Sato E, et al. Inhibition of binding of Helicobacter pylori to the glycolipid receptors by probiotic Lactobacillus reuteri. FEMS Immunol Med Microbiol. 2002;32(2):105-110

51. Cunningham-Rundles $S$, Ahrné $S$, Johann-Liang $R$, et al. Effect of probiotic bacteria on microbial host defense, growth, and immune function in human immunodeficiency virus type-1 infection. Nutr. 2011;3(12):1042-1070

52. Van Niekerk E, Kirsten G, Blaauw R, Nel D. Probiotics and necrtotizing enterocolitis in HIV-exposed premature infants. Stellenbosch: United South African Neonatal Association (USANA) Congress; 2013.

53. Deshpande GC, Rao SC, Keil AD, Patole SK. Evidence-based guidelines for use of probiotics in preterm neonates. BMC Med. 2011:9:92

54. Lin $\mathrm{HC}$, Su BH, Chen AC, et al. Oral probiotics reduce the incidence and severity of necrotizing enterocolitis in very low birth weight infants. Pediatrics. 2005;115(1):1-4.

55. Desfrere $L$, de Oliveira I, Goffinet $F$, et al. Increased incidence of necrotizing enterocolitis in premature infants born to HIV-positive mothers. AIDS. 2005:19(14):1487-1493.

56. Bertino E, Di Nicola P, Giuliani F, et al. Benefits of human milk in preterm infant feeding. Journal of Pediatric and Neonatal Individualized Medicine (JPNIM). 2012;1(1):19-24.

57. World Health Organization. HIV and infant feeding 2010: an updated framework for priority action. Geneva: WHO; 2012

58. Israel-Ballard K, Donovan R, Chantry C, et al. Flash-heat inactivation of HIV-1 in human milk: a potential method to reduce postnatal transmission in developing countries. $J$ Acquir Immune Defic Syndr. 2007:45(3):318-323.

59. Conde-Agudelo A, Belizán JM, Diaz-Rossello J. Kangaroo mother care to reduce morbidity and mortality in low birthweight infants. Cochrane Database Syst Rev. 2011;(3):CD002771
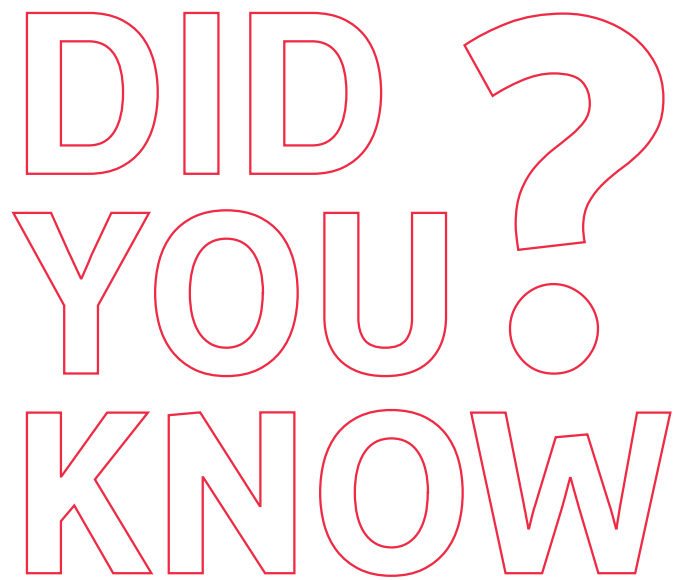

In South Africa:

2 in 5 females and 1 in 10 males are obese ${ }^{(1)}$

DIETARY ENERGY RESTRICTION OR PHYSICAL ACTIVITY IN THE MANAGEMENT OF OBESITY? IS THERE AN ANSWER? Yes, restriction of energy intake $\underline{\mathbf{P L U S}}$ exercise is more effective for weight loss than exercising or dieting independently. ${ }^{(2)}$

\section{PLAY AN ACTIVE ROLE}

\section{Access the science and expert opinions to debate the role of energy balance in the management of obesity.}

Visit http://beverageinstitute.org/southernafrica/landing_page/energy-balance/...today for more information on energy balance, physical activity and nutrition.

The Coca-Cola Company Beverage Institute for Health \& Wellness - Southern Africa, is a resource portal for scientific articles, expert opinions, tools and printable downloads to help manage the daily health, wellness and active living of your patients. We would also like to invite you to participate in our ongoing CPD Point Programmes, accredited by the Health Professions Council of South Africa.

References: 1. Human Sciences Research Council. The South African National Health and Nutrition Examination Survey. SANHANES-1. [Online] 2013 [cited 2014 Jul 9]. Available from: URL: http://www. hsrc.ac.za/uploads/pageNews/72/SANHANES-launch\%20edition\%20(online\%20version).pdf. 2. Lambert EV, Goedecke JH. Energy balance and energy expenditure in obesity - is obesity a disease of inactivity? Sports Med 2003:21-25. [Online] 2003 [cited 2014 Jul 23]. Available from: URL: http://www.ajol.info/index.php/sasma/article/viewFile/31880/23615. 